\title{
Dimensional reduction in Bose-Einstein condensed clouds of atoms confined in tight potentials of any geometry and any interaction strength
}

\author{
P. Sandin ${ }^{1}$, M. Ögren ${ }^{1}$, M. Gulliksson ${ }^{1}$ \\ ${ }^{1}$ School of Science and Technology, Örebro University, 70182 Örebro, Sweden \\ J. Smyrnakis ${ }^{2}$, M. Magiropoulos ${ }^{2}$, and G. M. Kavoulakis ${ }^{2}$ \\ ${ }^{2}$ Technological Education Institute of Crete, P.O. Box 1939, GR-71004, Heraklion, Greece
}

(Dated: November 10, 2018)

\begin{abstract}
Motivated by numerous experiments on Bose-Einstein condensed atoms which have been performed in tight trapping potentials of various geometries (elongated and/or toroidal/annular), we develop a general method which allows us to reduce the corresponding three-dimensional GrossPitaevskii equation for the order parameter into an effectively one-dimensional equation, taking into account the interactions (i.e., treating the width of the transverse profile variationally) and the curvature of the trapping potential. As an application of our model we consider atoms which rotate in a toroidal trapping potential. We evaluate the state of lowest energy for a fixed value of the angular momentum within various approximations of the effectively one-dimensional model and compare our results with the full solution of the three-dimensional problem, thus getting evidence for the accuracy of our model.

PACS numbers: 05.30.Jp, 67.85.Hj, 67.85.De, 03.75.Kk
\end{abstract}

\section{INTRODUCTION}

One of the many interesting and novel features of cold-atomic systems is the presence of a trapping potential. Compared to the homogeneous systems, these gases are finite, with a discrete energy spectrum. It is interesting that experimentalists can create trapping potentials which are both quasi-one- and quasi-two-dimensional, while more recently they have built annular and toroidal potentials. Clearly the reduced effective dimensionality of these systems, as well as the nontrivial topology of annular/toroidal traps introduce novel effects.

Quasi-one-dimensional traps have been used in cold atomic systems ever since the first pioneering experiments and have given rise to very interesting effects. Already almost 20 years ago the propagation of sound waves in an elongated Bose-Einstein condensate was observed and studied experimentally [1]. Soon after solitary waves were observed in an elongated trap [2] and in a more oblate one [3]. More recently toroidal and annular traps have been built, and even persistent currents have been observed, see, e.g., [4-12]. Remarkably, it has also become possible to manipulate the shape of the trapping potentials to a very high degree, see, e.g., Ref. [13].

Under typical conditions the non-linear Gross-Pitaevskii equation provides a very accurate description of these systems. In principle this equation may be solved numerically with use of various techniques, but still the full threedimensional problem may become rather challenging. When the trapping potential is very tight along two, or one dimension (i.e., the quantum of energy along the corresponding direction is much larger than any other energy scale of the problem) the motion of the atoms is quasi-one, or quasi-two-dimensional, respectively. In this case one may make an ansatz for the order parameter, assuming a decoupling of the degrees of freedom along the direction of tight confinement, which are frozen. Integrating over these degrees of freedom one may derive an effectively one-, or two-dimensional equation for the order parameter. Such efforts have been made by numerous authors. Given the large numbers of these references we refer just to some representative ones, [14 -18].

The benefit from such effective theories is two-fold. First of all, the corresponding numerical problem is easier to solve. Secondly, especially in the case of quasi-one-dimensional motion, one has a handy equation to work with, which may provide insight into the problem. In addition, one may derive simple expressions for various observables, thus making contact with the well-known corresponding expressions of the homogeneous systems (i.e., the one where there is no trapping potential).

Motivated by the numerous experiments which have been performed in such traps, as well as by the arguments presented above, we consider quite generally a tight, quasi-one-dimensional potential of any geometry and develop an effectively one-dimensional theory. This is the first main result of the present study. Then, we apply our model to the problem of atoms which rotate in a toroidal trapping potential. We solve numerically the one-dimensional problem under various approximations, i.e., the purely one-dimensional problem, the quasi-one-dimensional problem with a fixed transverse width, and the quasi-one-dimensional problem with the transverse width treated variationally. In addition we solve the full three-dimensional problem numerically, thus making a quantitative comparison of the various models with the exact solution, which is our second main result. 
In what follows below we present in Sec. II our quasi-one-dimensional model, while in Sec. III we give approximate and limiting expressions that result from it. In Sec. IV we show the corresponding numerical solutions that result within each approximation, as well as the results of the full three-dimensional problem and compare them. Finally in Sec. V we summarize our results and give an overview.

\section{DERIVATION OF THE EFFECTIVE 1D MODEL}

The starting point is the (time-independent) Gross-Pitaevskii equation in three dimensions,

$$
-\frac{1}{2} \nabla^{2} \Phi+V \Phi+N U_{0}|\Phi|^{2} \Phi-\mu \Phi=0
$$

where $\Phi$ is the order parameter, $N$ is the atom number, $V$ is the trapping potential, $U_{0}$ is the matrix element for atom-atom collisions, and $\mu$ is the chemical potential (the mass of the atoms $M$, as well as $\hbar$ are set equal to unity, while $\Phi$ is normalized to unity).

Let us now suppose that we want to solve the above three-dimensional Gross-Pitaevskii equation in a narrow tubular neighbourhood of a planar curve $\underline{x}_{0}(s)$. We use the following parametrization,

$$
\underline{x}(s, \rho, \phi)=\underline{x}_{0}(s)+\rho \sin \phi \hat{n}(s)+\rho \cos \phi \hat{n}(s) \times \hat{T}(s) .
$$

The schematic plot of Fig. 1 shows how $\rho, \phi$, and $s$ are defined. In Eq. (2) $\hat{T}(s)$ is the tangent and $\hat{n}(s)$ is the outer normal to the curve $\underline{x}_{0}(s)$. It is convenient to set $s$ equal to the arclength from a fixed origin of the curve. If we do this, $d \underline{x}_{0}(s) / d s=\hat{T}(s), d \hat{T}(s) / d s=-k(s) \hat{n}(s)$ and $d \hat{n}(s) / d s=k(s) \hat{T}(s)$, where $k(s)$ is the curvature of the curve. In the above coordinates the metric $d w^{2}$ becomes

$$
\begin{aligned}
d w^{2} & =d \underline{x} \cdot d \underline{x}=\left|\frac{d \underline{x}}{d s}\right|^{2} d s^{2}+\left|\frac{d \underline{x}}{d \rho}\right|^{2} d \rho^{2}+\left|\frac{d \underline{x}}{d \phi}\right|^{2} d \phi^{2} \\
& =[1+k(s) \rho \sin \phi]^{2} d s^{2}+d \rho^{2}+\rho^{2} d \phi^{2}
\end{aligned}
$$

Equation (11) thus becomes,

$$
\begin{aligned}
& -\frac{1}{2} \frac{1}{1+k(s) \rho \sin \phi}\left[\frac{\partial}{\partial s}\left(\frac{1}{1+k(s) \rho \sin \phi} \frac{\partial \Phi}{\partial s}\right)+\frac{1}{\rho} \frac{\partial}{\partial \rho}\left(\rho[1+k(s) \rho \sin \phi] \frac{\partial \Phi}{\partial \rho}\right)\right. \\
& \left.+\frac{1}{\rho} \frac{\partial}{\partial \phi}\left([1+k(s) \rho \sin \phi] \frac{\partial \Phi}{\rho \partial \phi}\right)\right]+V(\rho) \Phi+N U_{0}|\Phi|^{2} \Phi-\mu \Phi=0
\end{aligned}
$$

where the normalization for $\Phi$ has the form

$$
\int|\Phi|^{2}[1+k(s) \rho \sin \phi] \rho d \rho d s d \phi=1
$$

The trapping potential $V$ in the problem that we have in mind is assumed to act transversely to the curve and is a function of $\rho$ only, while it is taken to be harmonic, $V(\rho)=\omega^{2} \rho^{2} / 2$.

Setting $\Psi=\sqrt{h(\rho, s, \phi)} \Phi$, where $h \equiv 1+k(s) \rho \sin \phi$ we get for $\Psi$

$$
-\frac{1}{2} \frac{\partial}{\partial s}\left(\frac{\Psi_{s}}{h^{2}}\right)-\frac{1}{2} \frac{1}{\rho} \frac{\partial}{\partial \rho}\left(\rho \Psi_{\rho}\right)-\frac{1}{2} \frac{1}{\rho^{2}} \Psi_{\phi \phi}-\frac{1}{8} \frac{k^{2}(s)}{h^{2}} \Psi-\frac{5}{8} \frac{h_{s}^{2}}{h^{4}} \Psi+\frac{1}{4} \frac{h_{s s}}{h^{3}} \Psi+V(\rho) \Psi+N U_{0} h|\Psi|^{2} \Psi-\mu \Psi=0,
$$

where the low index on the right denotes differentiation with respect to the corresponding variable. The Hamiltonian that corresponds to Eq. (6) is

$$
\begin{array}{r}
H=\int\left[\frac{1}{2} \frac{\left|\Psi_{s}\right|^{2}}{h^{2}}+\frac{1}{2}\left|\Psi_{\rho}\right|^{2}+\frac{1}{2} \frac{1}{\rho^{2}}\left|\Psi_{\phi}\right|^{2}-\frac{1}{8} \frac{k^{2}(s)}{h^{2}}|\Psi|^{2}-\frac{5}{8} \frac{h_{s}^{2}}{h^{4}}|\Psi|^{2}+\frac{1}{4} \frac{h_{s s}}{h^{3}}|\Psi|^{2}\right. \\
\left.+V(\rho)|\Psi|^{2}+\frac{1}{2} \frac{1}{h} N U_{0}|\Psi|^{4}-\mu|\Psi|^{2}\right] \rho d \rho d s d \phi .
\end{array}
$$

Up to now the calculation we have performed is exact. In what follows below we will make the following ansatz

$$
\Psi(s, \rho, \phi)=\psi_{\operatorname{tr}}\left(\rho, a_{\perp}(s)\right) \cdot \psi(s),
$$




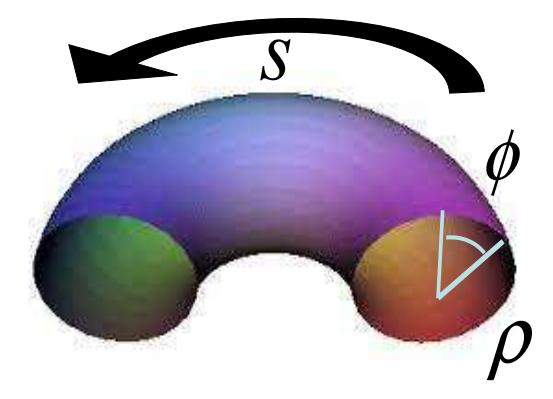

FIG. 1: (Colour online) Schematic picture, which shows the coordinates $\rho, \phi$, and $s$.

where

$$
\psi_{\operatorname{tr}}\left(\rho, a_{\perp}(s)\right)=\frac{1}{\sqrt{\pi} a_{\perp}(s)} e^{-\rho^{2} / 2 a_{\perp}^{2}(s)}
$$

is the normalized, rotationally symmetric, ground state of $V(\rho)$, with a spatially-dependent width $a_{\perp}(s)$, which is attributed to the (density-dependent) nonlinear term. The above ansatz assumes that the order parameter is independent of the azimuthal variable $\phi$. In addition, within this ansatz the functional form of the transverse profile of the cloud is Gaussian, however the cloud is allowed to expand radially, rather than having a fixed width, which would be the oscillator length $a_{0}=1 / \sqrt{\omega}$.

In Ref. [15] a closely-related problem has been solved, namely the one where there is no curvature, $k(s)=0$, making the same ansatz as that of Eq. (8). In the limit of weak interactions the actual transverse profile is Gaussian, while in the Thomas-Fermi limit the profile is an inverse parabola. Here, since we want our model to be applicable for any interaction strength we have chosen to work with a Gaussian for all interaction strengths, however its width $a_{\perp}$ is treated variationally and it is allowed to increase when the interaction is sufficiently strong. Therefore, viewed as a variational approach, the present model is expected to work rather well, as we confirm in Secs. III and IV.

The authors of Ref. [18] have attacked the same problem as the present one, including the effect of the curvature. However, in Ref. [18] the transverse profile has a fixed width, i.e., $a_{\perp}$ has been set equal to the oscillator length $a_{0}$. Thus, for zero, or weak interactions the two approaches coincide. However, our model is more general and it works better when the interaction becomes comparable to, or larger than, the oscillator quantum of energy $\omega$, as seen in the evaluation of the speed of sound in Sec. III and in the explicit problem considered in Sec. IV.

Performing the integration in Eq. (7) over the variable $\phi$, we find that

$$
\begin{aligned}
& H=\int\left[\frac{1}{2} \frac{1}{\left[1-k^{2}(s) \rho^{2}\right]^{3 / 2}}\left|\Psi_{s}\right|^{2}+\frac{1}{2}\left|\Psi_{\rho}\right|^{2}-\frac{1}{8} \frac{k^{2}(s)}{\left[1-k^{2}(s) \rho^{2}\right]^{3 / 2}}|\Psi|^{2}-\frac{5}{8} \frac{A(\rho, s)}{2 \pi}|\Psi|^{2}+\frac{1}{4} \frac{B(\rho, s)}{2 \pi}|\Psi|^{2}\right. \\
& \left.+\frac{1}{2} \frac{N U_{0}}{\sqrt{1-k^{2}(s) \rho^{2}}}|\Psi|^{4}+V(\rho)|\Psi|^{2}-\mu|\Psi|^{2}\right] 2 \pi \rho d \rho d s,
\end{aligned}
$$

where [19]

$$
A=-\left[k_{s}(s) \rho\right]^{2} \frac{3 \pi k(s) \rho}{\left[1-k^{2}(s) \rho^{2}\right]^{3 / 2}},
$$

and

$$
B=k_{s s}(s) \pi \rho \frac{1+4 k^{2}(s) \rho^{2}}{\left[1-k^{2}(s) \rho^{2}\right]^{7 / 2}} .
$$

Equation (10) leads to the $\phi$-independent equation

$$
\begin{array}{r}
-\frac{1}{2} \frac{\partial}{\partial s}\left[\frac{1}{\left[1-k^{2}(s) \rho^{2}\right]^{3 / 2}} \Psi_{s}\right]-\frac{1}{2} \frac{1}{\rho} \frac{\partial}{\partial \rho}\left(\rho \Psi_{\rho}\right)-\frac{1}{8} \frac{k^{2}(s)}{\left[1-k^{2}(s) \rho^{2}\right]^{3 / 2}} \Psi-\frac{5}{8} \frac{A(\rho, s)}{2 \pi} \Psi+\frac{1}{4} \frac{B(\rho, s)}{2 \pi} \Psi+ \\
+\frac{N U_{0}}{\sqrt{1-k^{2}(s) \rho^{2}}}|\Psi|^{2} \Psi+V(\rho) \Psi-\mu \Psi=0 .
\end{array}
$$


Up to now we have only made use of the independence of the order parameter from $\phi$ and not of the factorization in the form of a longitudinal and a transverse function.

Before we proceed, it is instructive to mention that there are three relevant length scales in the present problem, namely (i) the oscillator length $a_{0}$ - that sets the scale for the transverse width of the cloud $a_{\perp}$, (ii) the coherence (or healing) length $\xi$, that is defined as $1 /\left(2 \xi^{2}\right)=n_{0} U_{0}$, where $n_{0}$ is the three-dimensional density of the homogeneous system and it sets the scale of the solitary-wave, travelling wave solutions when the potential is sufficiently tight, and (iii) the length scale associated with the curvature, $k^{-1}$, that determines the scale over which the trapping potential "bends".

We now use the factorization made in the ansatz of Eq. (8), as well as Eq. (9). Furthermore, we assume that $a_{\perp} \ll\left|k^{-1}\right|$ and $a_{\perp} \ll \xi$. These conditions imply that $|k| a_{\perp} \ll 1$ and $\left(a_{\perp}\right)_{s} \ll 1$. Thus, we find the effectively one-dimensional Hamiltonian

$$
H_{1 d}=\int\left[\frac{1}{2}\left|\psi_{s}\right|^{2}+\frac{1}{2 a_{\perp}^{2}}|\psi|^{2}-\frac{1}{8} k^{2}(s)|\psi|^{2}+\frac{1}{2} \frac{N U_{0}}{2 \pi a_{\perp}^{2}}|\psi|^{4}+\frac{1}{2} \omega^{2} a_{\perp}^{2}|\psi|^{2}-\mu|\psi|^{2}\right] d s,
$$

where $\int|\psi|^{2} d s=1$. Demanding that variations in $\psi^{*}$ and in $a_{\perp}^{2}$ in the above functional vanish we find that

$$
-\frac{1}{2} \psi_{s s}+\frac{1}{2 a_{\perp}^{2}} \psi-\frac{1}{8} k^{2}(s) \psi+\frac{N U_{0}}{2 \pi a_{\perp}^{2}}|\psi|^{2} \psi+\frac{1}{2} \omega^{2} a_{\perp}^{2} \psi-\mu \psi=0
$$

and

$$
a_{\perp}^{4}=\frac{1}{\omega^{2}}\left[1+\frac{N U_{0}|\psi|^{2}}{2 \pi}\right]=a_{0}^{4}\left[1+\frac{N U_{0}|\psi|^{2}}{2 \pi}\right] .
$$

The coefficient of the nonlinear term in Eq. (15) may be identified as the integral of $\left|\psi_{\operatorname{tr}}(\rho)\right|^{4}$ over the cross section of the cloud, $U_{0} \int\left|\psi_{\operatorname{tr}}(\rho)\right|^{4} \rho d \rho d \phi=U_{0} /\left(2 \pi a_{\perp}^{2}\right)$. The dimensionless quantity $N U_{0}|\psi|^{2} /(2 \pi)$ in Eq. (16) is equal to the ratio between the interaction energy and the quantum of energy $\omega$. If $a_{\mathrm{sc}}$ is the scattering length for elastic atom-atom collisions, then $U_{0}=4 \pi a_{\mathrm{sc}}$, and therefore $N U_{0}|\psi|^{2} /(2 \pi)=2 \sigma a_{\mathrm{sc}}$, where $\sigma \equiv N|\psi|^{2}$ is the density per unit length.

Combining Eqs. (15) and (16) we get that

$$
-\frac{1}{2} \psi_{s s}+\left[\frac{\omega}{2 \sqrt{1+N U_{0}|\psi|^{2} /(2 \pi)}}-\frac{1}{8} k^{2}(s)+\frac{1}{2} \omega \sqrt{1+N U_{0}|\psi|^{2} /(2 \pi)}+\frac{N U_{0} \omega}{2 \pi \sqrt{1+N U_{0}|\psi|^{2} /(2 \pi)}}|\psi|^{2}-\mu\right] \psi=0 .(17
$$

The above equation is the first main result of the present study. The initial problem, which has three spatial dimensions, has been reduced to a problem of one dimension. We stress the generality of the above equation, where for some given $k(s)$ - i.e., a trapping geometry of any shape - and also for any interaction strength, these equations are applicable (under the assumptions that we mentioned above). In addition, it is interesting that one may identify separately the effect of the curvature, of the transverse confinement, and of the interaction.

\section{APPROXIMATIONS AND APPLICATIONS OF THE EFFECTIVE 1D MODEL}

It is instructive to examine some limiting cases of our model, which also allows us to get some insight. First of all, Eq. (16) is an algebraic equation for the transverse width of the cloud, $a_{\perp}$. In the absence of interactions $a_{\perp}$ is equal to the oscillator length $a_{0}$, as expected. When the system is in the Thomas-Fermi regime, i.e., the interaction energy is much larger than $\omega$, then $a_{\perp} / a_{0} \approx\left(2 \sigma a_{\mathrm{sc}}\right)^{1 / 4}$. In this limit the width of the cloud thus increases due to the interactions [14, 15], since $\sigma a_{\mathrm{sc}} \gg 1$. We should stress that in the Thomas-Fermi limit the coherence length may become smaller than the oscillator length and our model approaches its limits of validity [15].

Equation (17) has various interesting limits. For weak interactions [18],

$$
-\frac{1}{2} \psi_{s s}+\left[-\frac{1}{8} k^{2}(s)+\frac{N U_{0}}{2 \pi a_{0}^{2}}|\psi|^{2}+\omega-\mu\right] \psi=0 .
$$

In the case of a ring-like potential, where $k(s)$ is constant and equal to $1 / R$, with $R$ being the radius of the ring, the effect of the curvature is trivial, since the above equation essentially coincides with the purely one-dimensional equation.

In the opposite limit of "strong" interactions, i.e., in the Thomas-Fermi limit the nonlinear term in the effective equation, Eq. (17), has a different functional form, which is $|\psi| \psi$, instead of the usual one, $|\psi|^{2} \psi$ [15],

$$
-\frac{1}{2} \psi_{s s}+\left[-\frac{1}{8} k^{2}(s)+\frac{3}{2} \omega \sqrt{\frac{N U_{0}}{2 \pi}}|\psi|-\mu\right] \psi=0 .
$$


Setting $k(s)=0$ and after linearisation one gets the following Bogoliubov spectrum from Eq. (19)

$$
E(p)=\sqrt{\frac{1}{4} p^{4}+\frac{3}{4}\left(\frac{\sigma_{0} U_{0}}{2 \pi}\right)^{1 / 2} \omega p^{2}},
$$

for the energy $E$, where $p$ is the momentum and $\sigma_{0}$ is the background, one-dimensional density, i.e., the density at $|s| \rightarrow \infty$. For $p \rightarrow 0$ we get a speed of sound which is

$$
c^{2}=\frac{3}{4} \omega \sqrt{\frac{\sigma_{0} U_{0}}{2 \pi}}=\frac{3 \sqrt{2}}{4} \omega \sqrt{\sigma_{0} a_{\mathrm{sc}}} .
$$

The above expression should be compared with the exact result [15] (i.e., the one with the inverse parabola for the transverse profile, instead of the Gaussian that is assumed here),

$$
c_{\mathrm{ex}}^{2}=\omega \sqrt{\frac{\sigma_{0} U_{0}}{4 \pi}}=\omega \sqrt{\sigma_{0} a_{\mathrm{sc}}}
$$

and thus $\left(c / c_{\mathrm{ex}}\right)^{2}=(3 \sqrt{2} / 4) \approx 1.06$. As expected, the speed of sound in the approximate scheme is higher, however the difference (roughly $3 \%$ for the velocities) is rather small. On the other hand, if one uses Eq. (18) instead (thus ignoring the effect of the interaction on the width of the transverse profile), then $\left(c / c_{\mathrm{ex}}\right)^{2}=2 \sqrt{\sigma_{0} a_{\mathrm{sc}}}$, which is $\gg 1$ in the Thomas-Fermi limit, and thus this model fails.

\section{NUMERICAL RESULTS}

\section{A. Bosonic atoms confined in a tight toroidal trap}

Having established the general framework which allows us to reduce the three-dimensional problem into an effectively one-dimensional equation, we now apply our method to the problem of a toroidal trapping potential, and in particular to the problem where the atoms are given some angular momentum. In the case of an axially-symmetric (toroidal) geometry $k(s)=1 / R$. To make a comparison between the various models, first of all, we solve the purely onedimensional problem,

$$
-\frac{1}{2 R^{2}} \frac{d^{2} \psi}{d \theta^{2}}+\left[\frac{N U_{0}}{2 \pi a_{0}^{2}}|\psi|^{2}+\omega-\mu\right] \psi+i \Omega \frac{d \psi}{d \theta}=0 .
$$

Here $\Omega$ is a Lagrange multiplier that takes care of the angular momentum and $\theta=s / R$. This equation is solved under the constraints of a fixed atom number and a fixed value of the angular momentum; for more details see Ref. [20].

We also solve the same problem (i.e., under the same constraints of a fixed atom number and a fixed value of the angular momentum, with $k(s)=1 / R$ ) using Eq. (15), setting the transverse width $a_{\perp}$ equal to $a_{0}$. As we mentioned earlier, this approximation for the transverse width being constant and equal to $a_{0}$ has been made in Ref. [18]. Finally, we solve the same problem using Eqs. (15) and (16), i.e., we solve Eq. (17), thus treating the transverse width of the cloud $a_{\perp}$ variationally.

The plots in Fig. 2 show the dispersion relation within (i) the strictly one-dimensional model, (ii) the effective one-dimensional model with a fixed transverse width (corresponding to the calculation of Ref. [18]), (iii) the effective one-dimensional model with a variable transverse width, and finally (iv) the energy of the full three-dimensional solutions, for three values of $\omega$ and $U_{0}$ (as explained in detail in the following section). The energy between the first and the second differ by the constant factor $\Delta E=-k^{2} / 8=-1 / 8$ (for $R=1$ that we have assumed) due to the constant curvature term in the energy. As the ratio $n_{0} U_{0} / \omega$ decreases the system approaches the one-dimensional limit. Indeed, as seen from these three figures, as this ratio decreases all the curves come closer to each other. More importantly, for the largest ratio of $n_{0} U_{0} / \omega$, where there are substantial deviations from the one-dimensional limit, our model with a variable width provides an accurate description of the energy of the full, three-dimensional problem.

The plots in Fig. 3 show the density of the order parameter for two values of the angular momentum $\ell=1 / 2$ and $\ell=3 / 4$. In order for the effect of the interaction to become more pronounced, we set the ratio $n_{0} U_{0} / \omega=5 / 2$. For this choice of parameters, $\xi / a_{0}=1 / \sqrt{5} \approx 0.447, R / a_{0}=\sqrt{20} \approx 4.472$, while $\xi / R=1 / 10$. Within the purely one-dimensional model the solution with $\ell=1 / 2$ corresponds to a "dark" solitary wave and thus there is a node in the density, as seen in Fig. 5. This feature is preserved within our quasi-one-dimensional model with a variable transverse width. In addition, for both values of $\ell$ we observe that the width of this solution is larger than the one of the purely one-dimensional model. 

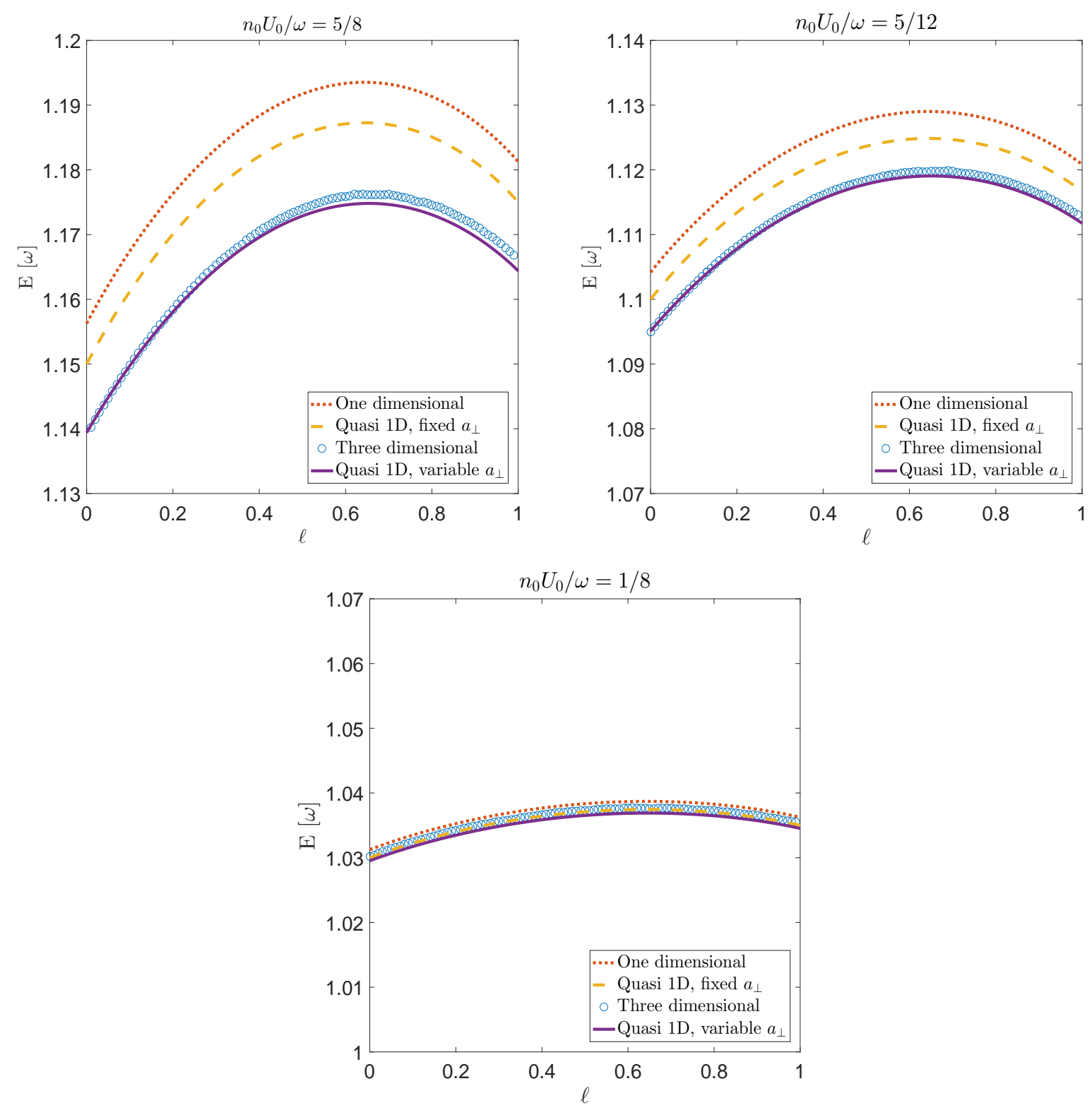

FIG. 2: (Colour online) The dispersion relation, i.e., the energy versus the angular momentum per particle $\ell$, (i) within the strictly one-dimensional model, (ii) within the effective one-dimensional model with a fixed transverse width (corresponding to the calculation of Ref. [18]), (iii) within the effective one-dimensional model with a variable transverse width, and finally (iv) within the full, three-dimensional problem. Here $R / \xi=5$ in all the plots, and also $\xi / a_{0}=2 / \sqrt{5} \approx 0.894, R / a_{0}=\sqrt{20} \approx 4.472$ (upper left); $\xi / a_{0}=1.095, R / a_{0}=\sqrt{30} \approx 5.477$ (upper right); and $\xi / a_{0}=2, R / a_{0}=10$ (lower).

\section{B. Full, three-dimensional problem and comparison with the effective one-dimensional models}

To make a comparison with an "exact" problem, we also solve the full, three-dimensional problem. To do this, we consider a trapping potential that has the form (in cylindrical coordinates)

$$
V(\rho, \phi, z)=\frac{1}{2} \omega^{2}\left[(\rho-R)^{2}+z^{2}\right] .
$$

We impose the same constraints as above, namely a fixed atom number and a fixed value of the angular momentum.

Since one of the main purposes of our study is to identify the effect of the deviations from purely one-dimensional motion, first of all, we need to identify this limit. This limit is achieved when the interaction energy $n_{0} U_{0}$ is much 

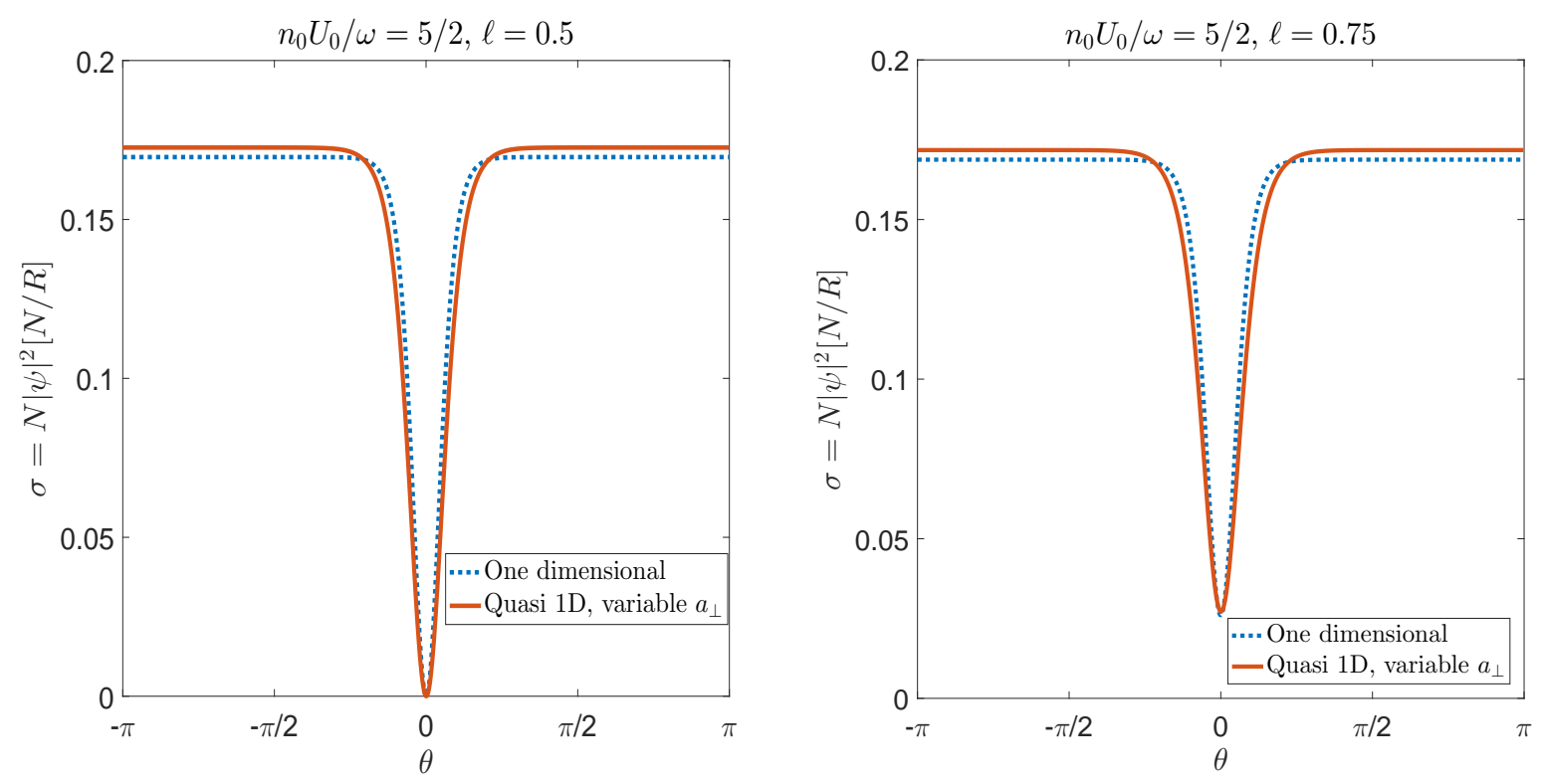

FIG. 3: (Colour online) The density per unit length $\sigma=N|\psi|^{2}$ of the order parameter $\psi$ as function of the angle $\theta$ within the purely one-dimensional model, and within the effective one-dimensional model with a variable transverse width for $\xi / a_{0}=$ $1 / \sqrt{5} \approx 0.447, R / a_{0}=\sqrt{20} \approx 4.472$, and $\xi / R=1 / 10$, for $\ell=1 / 2$ (left), and $\ell=3 / 4$ (right).

less that $\omega$. For weak interactions, $n_{0}=N /\left(2 \pi R \pi a_{0}^{2}\right)$, and thus $n_{0} U_{0} / \omega=N U_{0} /\left(2 \pi^{2} R\right)$. We stress that this ratio does not depend on $\omega$, since the density scales as $a_{0}^{-2}=\omega$. Finally, the corresponding ratio $\xi / a_{0}$ is $\sqrt{\pi^{2} R /\left(N U_{0}\right)}$.

The other relevant ratio is that between $n_{0} U_{0}$ and the kinetic energy $K$ for motion of the atoms along a ring with radius $R$, i.e., $K=1 /\left(2 R^{2}\right)$. The ratio $n_{0} U_{0} / K$ is given by $N U_{0} \omega R / \pi^{2}$. In addition, $\xi / R=\pi / \sqrt{N U_{0} \omega R}$. For fixed $N$ and $R$, the limit of one-dimensional motion is the one where $U_{0}$ is "small" (in the sense described above), with $n_{0} U_{0} / K=N U_{0} \omega R / \pi^{2}$ fixed, which implies that the product $U_{0} \omega$ has to be kept fixed.

From the arguments described above, we choose three sets of parameters. In all of them we set $n_{0} U_{0} / K=25$, or in terms of length scales $\xi / R=1 / 5$. In the first set $n_{0} U_{0} / \omega=5 / 8=0.625$, or in terms of length scales $\xi / a_{0}=2 / \sqrt{5} \approx 0.894$, while $R / a_{0}=\sqrt{20} \approx 4.472$. In the second intermediate set the system is closer to onedimensional motion. Here, $n_{0} U_{0} / \omega=5 / 12 \approx 0.417$, or $\xi / a_{0}=1.095$, and $R / a_{0}=\sqrt{30} \approx 5.477$. Finally, in the third set we are even closer to the one-dimensional limit, $n_{0} U_{0} / \omega=1 / 8=0.125$, or in terms of length scales $\xi / a_{0}=2$, while $R / a_{0}=10$. In the first two cases the coherence length is roughly equal with the oscillator length, while in the third it is twice as large.

Especially in the first case the transverse degrees of freedom start to play a role. We stress that the condition for quasi-one-dimensional motion implies that the interaction energy does not exceed the oscillator quantum of energy in the transverse direction (an equivalent way of expressing this condition is that the coherence length, which sets the scale of the width of the solitary-wave profiles, is at least on the order of, or larger than the transverse width of the cloud, which is set by the oscillator length.) Still, even in the first case, where we have come closer to breaking the limits of validity of our model, we see in Fig. 2 that our results are rather accurate.

The coupled three-dimensional equations are solved using the built-in stationary solver in the commercial software COMSOL Multiphysics, version 5.2a. The domain is set up as a torus with major radius $R=1$, and minor radius $4 a_{0}=4 / \sqrt{\omega}$. This minor radius of the computational domain is deemed sufficiently large to ignore boundary effects. Neumann boundary conditions are used and the equations are solved on a tetrahedral mesh. After having studied the convergence for different number of finite elements, we finally used a mesh with about $10^{5}$ elements. The exact number of elements differ depending on the size of the domain, determined by the value of $\omega$.

Initial data that is not too far away from the desired solution is needed to find a solution of the nonlinear equations. To overcome this difficulty we take advantage of the one-dimensional solutions and "dress" them with a Gaussian profile in the transverse direction to obtain initial data good enough to find a first solution. The equations are then solved in sequence of increasing angular momentum, where each solution is provided as initial data for the next run. For each run the constant determining the wanted angular momentum is increased with a variable step with a minimal value of 0.001 , until the full range $0 \leq \ell<1$ is covered. A subset of 100 solutions of the total set of solutions is then 


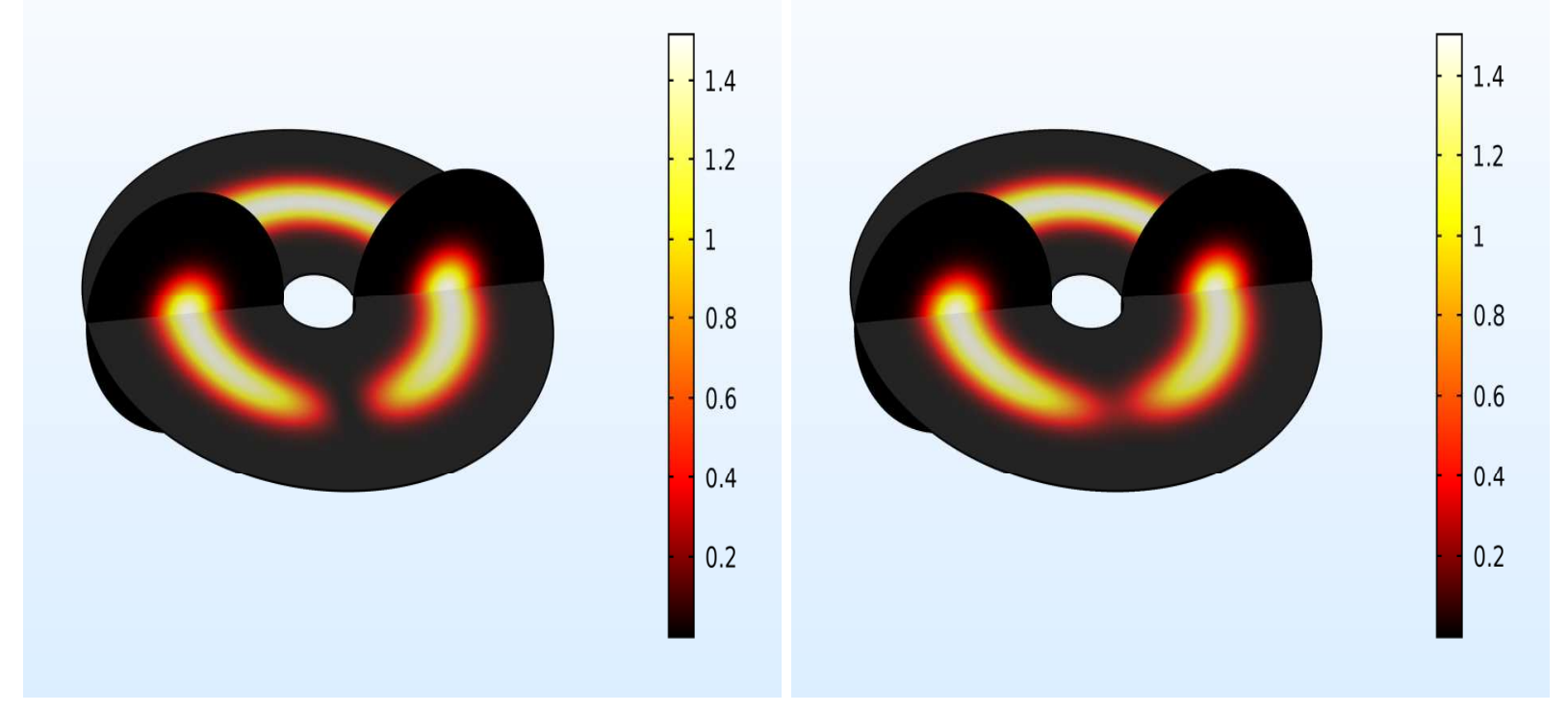

FIG. 4: (Colour online) The density $|\Phi|^{2}$ of the order parameter $\Phi$ of the full, three-dimensional model, on the $z=0$ plane, as well as along a perpendicular plane, for the same parameters as in the intermediate plot of Fig. 2, i.e., $n_{0} U_{0} / \omega=5 / 12$, $\xi / R=1 / 5, \xi / a_{0}=1.095$ and $R / a_{0}=\sqrt{30} \approx 5.477$. Here $\ell=1 / 2$ (left) and $\ell=3 / 4$ (right).

saved.

The plots in Fig. 4 show the density of the order parameter that is evaluated within the full, three-dimensional problem, for the same parameters as those of the intermediate plot of Fig. 2, i.e., $n_{0} U_{0} / \omega=5 / 12(\xi / R=1 / 5$, $\xi / a_{0}=1.095, R / a_{0}=\sqrt{30} \approx 5.477$ ), and for $\ell=1 / 2$ and $3 / 4$. For $\ell=1 / 2$ the density minimum is very low, which is a remnant of the "dark" solitary wave of the one-dimensional/quasi-one-dimensional models. For $\ell=3 / 4$, the density minimum is not as pronounced, again in agreement with the purely one-dimensional model, where the wave is "grey", instead of "dark", i.e., the density has a minimum which does not extend all the way to zero, though.

\section{SUMMARY AND CONCLUSIONS}

The high degree of tunability of cold atomic systems allows us to build a superfluid system with properties which are designed at will. Tuning the trapping potential allows us to restrict the motion of the atoms either in two, or even in one spatial dimension and also to build topologically non-trivial traps. In the present study we have developed a model which is suitable in the case of a relatively tight confinement in two spatial dimensions, which results into quasi-onedimensional motion. Apart from the assumption of relative tight confinement, it makes no further assumptions, and it manages to reduce the three-dimensional problem into an effective one-dimensional, for any geometry, all the way between weak to strong (provided, of course that the mean-field approximation is still valid) interatomic interactions.

The model that we have derived is useful for practical purposes, since it reduces the computational effort that is required in order to solve the corresponding nonlinear equation. Furthermore, our approach allows one to decouple and identify the three basic effects that enter this problem, namely the effect of the transverse confinement, of the curvature and of the interaction and see how each of them affects the system. Therefore it also provides insight into how all these various mechanisms affect the system, often in a competing way.

The quantitative comparison that we have made with the full numerical solution that we have found of the threedimensional problem provides strong evidence that our model is rather accurate. While in the present study we have restricted ourselves to the case of the lowest mode in the transverse direction, one may generalize this method in order to include more modes. Such an approach would be even more accurate and it may be applicable in problems where the deviations from purely one-dimensional motion are substantial. As a result, it would be suitable in describing instabilities because of the deviations from purely one-dimensional motion see, e.g., Refs. [21].

A whole new field, the so-called "atomtronics", which focuses on the creation of atomic analogues to electronic devices has started to develop, see, e.g., [22]. These experiments are deeply in the Thomas-Fermi regime with respect 
to the transverse degrees of freedom of the condensate. Developing models like the one presented here is certainly helpful, since they provide insight into these problems and also a relatively simple theoretical description. Another activity which is equally interesting is that of atomic waveguides (see, e.g., [23]), with obvious potential technological applications. Our model is applicable in such systems and it becomes especially interesting in the case where these waveguides bend, since it combines the effect of the curvature with that of the interaction.

Finally, the present model is applicable in other quasi-one-dimensional systems. For example, considering the effect of the curvature combined with dipolar interatomic interactions 24] (instead of contact, considered here), may give rise to interesting effects. Equally interesting and important may also be the study of the combined effect of curvature with spin-orbit coupling [25], which has been investigated in recent experiments. 
Acknowledgements. We acknowledge support from Örebro University, School of Science and Technology, and partly through RR 2015/2016.

[1] M. R. Andrews, D. M. Kurn, H.-J. Miesner, D. S. Durfee, C. G. Townsend, S. Inouye, and W. Ketterle, Phys. Rev. Lett. 79, 553 (1997).

[2] S. Burger, K. Bongs, S. Dettmer, W. Ertmer, K. Sengstock, A. Sanpera, G. V. Shlyapnikov, and M. Lewenstein, Phys. Rev. Lett. 83, 5198 (1999).

[3] J. Denschlag, J. E. Simsarian, D. L. Feder, Charles W. Clark, L. A. Collins, J. Cubizolles, L. Deng, E. W. Hagley, K. Helmerson, W. P. Reinhardt, S. L. Rolston, B. I. Schneider, and W. D. Phillips, Science 287, 97 (2000).

[4] S. Gupta, K. W. Murch, K. L. Moore, T. P. Purdy, and D. M. Stamper-Kurn, Phys. Rev. Lett. 95, 143201 (2005).

[5] Spencer E. Olson, Matthew L. Terraciano, Mark Bashkansky, and Fredrik K. Fatemi, Phys. Rev. A 76, 061404(R) (2007).

[6] C. Ryu, M. F. Andersen, P. Cladé, Vasant Natarajan, K. Helmerson, and W. D. Phillips, Phys. Rev. Lett. 99, 260401 (2007).

[7] B. E. Sherlock, M. Gildemeister, E. Owen, E. Nugent, and C. J. Foot, Phys. Rev. A 83, 043408 (2011).

[8] A. Ramanathan, K. C. Wright, S. R. Muniz, M. Zelan, W. T. Hill, C. J. Lobb, K. Helmerson, W. D. Phillips, and G. K. Campbell, Phys. Rev. Lett. 106, 130401 (2011).

[9] Stuart Moulder, Scott Beattie, Robert P. Smith, Naaman Tammuz, and Zoran Hadzibabic, Phys. Rev. A 86, 013629 (2012).

[10] C. Ryu, K. C. Henderson, and M. G. Boshier, New J. Phys. 16, 013046 (2014).

[11] Scott Beattie, Stuart Moulder, Richard J. Fletcher, and Zoran Hadzibabic, Phys. Rev. Lett. 110, 025301 (2013).

[12] Stephen Eckel, Jeffrey G. Lee, Fred Jendrzejewski, Noel Murray, Charles W. Clark, Christopher J. Lobb, William D. Phillips, Mark Edwards, and Gretchen K. Campbell, Nature (London) 506, 200 (2014).

[13] K. Henderson, C. Ryu, C. MacCormick, and M. G. Boshier, New Journal of Phys. 11, 043030 (2009).

[14] G. M. Kavoulakis and C. J. Pethick, Phys. Rev. A 58, 1563 (1998).

[15] A. D. Jackson, G. M. Kavoulakis, and C. J. Pethick, Phys. Rev. A, 58, 2417 (1998).

[16] P. Leboeuf and N. Pavloff, Phys. Rev. A 64, 033602 (2001).

[17] L. Salasnich, A. Parola, and L. Reatto, Phys. Rev. A 65, 043614 (2002).

[18] S. Schwartz, M. Cozzini, C. Menotti, I. Carusotto, P. Bouyer, and S. Stringari, New Journal of Phys. 8, 162 (2006).

[19] To evaluate the integrals $B$ and $A$ respectively, we start with the integral $I(a)=\int_{0}^{2 \pi} d \phi /(1+a \sin \phi)^{2}$, where $a=\kappa \rho$, and differentiate once and twice with respect to $a$.

[20] P. Sandin, M. Ögren, and M. Gulliksson, Phys. Rev. E 93, 033301 (2016).

[21] P. O. Fedichev, A. E. Muryshev, and G. V. Shlyapnikov, Phys. Rev. A 60, 3220 (1999); D. L. Feder, M. S. Pindzola, L. A. Collins, B. I. Schneider, and C. W. Clark Phys. Rev. A 62, 053606 (2000); S. Komineas and N. Papanicolaou Phys. Rev. Lett. 89, 070402 (2002).

[22] Ranchu Mathew, Avinash Kumar, Stephen Eckel, Fred Jendrzejewski, Gretchen K. Campbell, Mark Edwards,and Eite Tiesinga, Phys. Rev. A 92, 033602 (2015).

[23] Giovanni Luca Gattobigio, Antoine Couvert, Gael Reinaudi, Bertrand Georgeot, and David Guery-Odelin, Phys. Rev. Lett. 109, 030403 (2012).

[24] F. Malet, G. M. Kavoulakis, and S. M. Reimann, Phys. Rev. A 84, 043626 (2011).

[25] E. Ö. Karabulut, F. Malet, A. L. Fetter, G. M. Kavoulakis, and S. M. Reimann, New Journal of Phys. 18, 015013 (2016). 